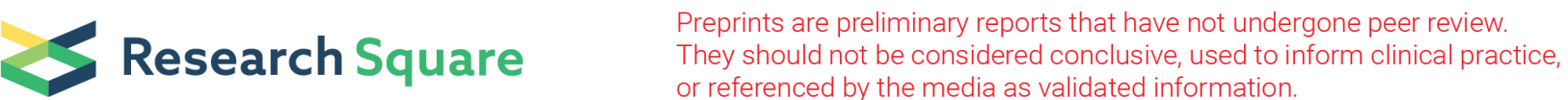

\section{Poor bone quality in patients with rheumatoid arthritis receiving long-term bisphosphonate therapy for glucocorticoid-induced osteoporosis: an iliac bone biopsy study}

Masahiko Takahata ( $\nabla$ takamasa@med.hokudai.ac.jp )

Hokkaido University https://orcid.org/0000-0002-2436-2175

Tomohiro Shimizu

Hokkaido University: Hokkaido Daigaku

Satoshi Yamada

Hokkaido University: Hokkaido Daigaku

Tomomaya Yamamoto

Hokkaido University: Hokkaido Daigaku

Tomoka Hasegawa

Hokkaido University: Hokkaido Daigaku

Ryo Fujita

Hokkaido University: Hokkaido Daigaku

Hideyuki Kobayashi

Hokkaido University: Hokkaido Daigaku

Tsutomu Endo

Hokkaido University: Hokkaido Daigaku

Yoshinao Koike

Hokkaido University: Hokkaido Daigaku

Norio Amizuka

Hokkaido University: Hokkaido Daigaku

Masahiro Todoh

Hokkaido University: Hokkaido Daigaku

Jun-ichiro Okumura

Sapporo City General Hospital: Shiritsu Sapporo Byoin

Tomomichi Kajino

Tonan hospital

Norimasa Iwasaki

Hokkaido University: Hokkaido Daigaku 
Research article

Keywords: rheumatoid arthritis, glucocorticoid-induced osteoporosis, bisphosphonate, bone quality, bone fragility

Posted Date: November 17th, 2021

DOI: https://doi.org/10.21203/rs.3.rs-1075694/v1

License: (c) (i) This work is licensed under a Creative Commons Attribution 4.0 International License.

Read Full License 


\section{Abstract \\ Background}

Bisphosphonates (BPs) have been shown to reduce the incidence of vertebral fractures during the first year or two of glucocorticoid (GC) treatments and are therefore recommended as a first-line treatment for the prevention and treatment of GC-induced osteoporosis (GIO). However, there are theoretical concerns about the long-term use of BPs in low-turnover osteoporosis caused by chronic GC therapy.

\section{Methods}

We analyzed the trabecular microarchitecture, bone metabolism, and material strength of bone biopsy samples from the iliac crest of female patients with rheumatoid arthritis (RA) receiving an average of 6.7 years of BP therapy for GIO (GIOBP group, $n=10$ ), compared with those of age- and bone mineral density (BMD)-matched non-RA postmenopausal women with no history of anti-osteoporosis medication use (reference group, $\mathrm{n}=10$ ).

\section{Results}

Patients in the GIOBP group had a significantly greater fracture severity index, as calculated by the sum of the Genant visual semiquantitative grading scores of vertebral fractures (T4-L5) compared with the reference patients. Micro-computed tomography analysis showed that the degree of mineralization and trabecular microarchitecture were significantly lower in the GIOBP group than in the BMD-matched reference patients. Patients in the GIOBP group exhibited lower bone contact stiffness, determined by micro-indentation testing, than in the reference group. The contact stiffness of the bone was negatively correlated with the fracture severity index and the daily prednisolone dosage. Immunohistochemistry and serum bone turnover markers showed decreased osteoclastic activity, impaired mineralization, and an increased fraction of empty lacunae in the GIOBP group.

\section{Conclusions}

Our findings indicate that patients with RA receiving long-term BP for GIO are still at high risk for fragility fractures because of poor bone quality.

\section{Background}

Despite profound advances in the treatment of rheumatic diseases, such as the development of biological agents, many patients with rheumatic diseases still receive glucocorticoid (GC) therapy to ease the symptoms of inflammation. While GC therapy effectively alleviates inflammation, it has a devastating 
effect on bone, leading to an increase in fragility fractures with an annual incidence of $3-16 \%$ in chronic GC users[1, 2].

In 2017 the American College of Rheumatology recommended bisphosphonates (BPs), inhibitors of osteoclast-mediated bone resorption, as the first-line drug for GC-induced osteoporosis because of their effectiveness, safety, low cost, and the lack of evidence of superior anti-fracture benefits conferred by other osteoporosis medications[3]. Several clinical trials for GC-induced osteoporosis (GIO) have demonstrated that BPs prevent bone loss and reduce the incidence of vertebral fractures during the first year or two of GC treatments [4-7]. However, the long-term efficacy of BP therapy for GIO remains uncertain[8, 9].

In contrast to postmenopausal osteoporosis, which causes high turnover osteoporosis, chronic GC therapy is known to induce low turnover osteoporosis, theoretically less effectively treated by antiresorptive therapy such as BP. Upon the initiation of GC therapy, bone resorption is transiently accelerated, yielding a rapid increase in bone fragility [9-13]. Hence, it is reasonable to administer antiresorptive agents such as BPs to prevent and treat GIO during the first year or two of GC treatments.

However, osteoclast activity changes from accelerated to downregulated in the chronic stage of GC therapy. Chronic GC therapy induces low-turnover osteoporosis because bone formation is constantly inhibited during GC treatment by impairing osteoblast proliferation and function [10-12, 14]. Therefore, BP therapy may further suppress bone remodeling, resulting in adynamic bone and compromised bone quality in patients receiving chronic GC therapy.

\section{Methods}

The purpose of this study was to characterize the bone quality features of patients receiving long-term BP therapy in the chronic stage of GC treatment. To achieve this, we analyzed the trabecular microarchitecture, bone metabolism, and material strength of bone biopsy samples from the iliac crest of female patients with rheumatoid arthritis (RA) receiving long-term BP therapy for GIO with reference to those of age- and bone mineral density (BMD)-matched non-RA postmenopausal women.

\section{Patients}

This study was approved by the Hokkaido University Hospital Institutional Review Board (\#012-0023). Written informed consent was obtained from all participants. For this observational study, 10 female Japanese patients with RA receiving GC and BP therapies for longer than 3 years and undergoing spine surgery with autologous iliac bone graft were recruited (BP therapy for GIO [GIOBP] group). All patients fulfilled the 2010 American College of Rheumatology/European League Against Rheumatism classification criteria for RA $[18,19]$ and were ambulatory at the time of the study.

The exclusion criteria were as follows: history of metabolic disorder that could affect bone metabolism (such as severe renal impairment defined as a creatinine clearance of less than $30 \mathrm{~mL} / \mathrm{min}$ ), thyroid or 
parathyroid disease, and malignancy. Ten age- and BMD-matched female patients who underwent spine surgery with an autologous iliac bone graft were recruited (reference group). All eligible participants in the reference group were as follows: 1) ambulatory, 2) without a history of autoimmune, thyroid or parathyroid disease, malignancy, or any other significant medical problems that required long-term treatment, with the exception of hypertension, type 2 diabetes mellitus (T2D), and dyslipidemia (under control with or without treatment), and 3) without a history of osteoporosis medication use.

\section{Demographic data}

The following data were collected from all patients: age, height, and weight (from which body mass index [BMI] was calculated); a complete medical history, including age at menopause, parental history of hip fracture, alcohol use, GC use, conventional and biological disease-modifying antirheumatic drug use, smoking, and current medication use. The 10-year probabilities for major osteoporotic and hip fractures were calculated using the World Health Organization Fracture Risk Assessment Tool (FRAX) [20].

\section{Serologic examination, dual-energy X-ray absorptiometry (DXA) testing, and assessment of existing vertebral fractures}

Blood sampling was performed preoperatively and included renal function, electrolytes, and bone metabolic markers, including bone-specific alkaline phosphatase (BAP) and tartrate-resistant acid phosphatase $5 \mathrm{~b}$ (TRACP-5b). BMD ( $\left.\mathrm{g} / \mathrm{cm}^{2}\right)$ was measured at the spine ( $\left.\mathrm{L} 2-\mathrm{L} 4\right)$ and left femoral neck by DXA (Discovery A, Hologic Japan, Inc, Tokyo, Japan). The number and severity of vertebral fractures were evaluated by a semiquantitative technique using lateral plain radiographs of the whole spine[15].

The vertebral fracture severity index, which we defined as an integrated value of semiquantitative grades of vertebral fractures, was calculated for each patient by two orthopedic surgeons[16]. For example, if a patient had two mild vertebral fractures (grade 1), one moderate vertebral fracture (grade 2), and one severe fracture (grade 3), the fracture severity was calculated as $2 \times 1+1 \times 2+1 \times 3=7$.

\section{Bone specimen collection}

Bone biopsy was performed during the autologous bone graft harvest from the posterior iliac crest. Bone specimens $(10 \times 10 \times 10 \mathrm{~mm})$ were harvested and subjected to radiographic analysis. After collecting three-dimensional structure data by micro-computed tomography (micro-CT), the specimens were divided into two pieces and subjected to mechanical testing and histological observation.

\section{Micro-CT bone structure analysis}

The specimens were scanned using micro-CT (R_mCT2; Rigaku, Tokyo, Japan) at a 20- $\mu$ m isotropic resolution. Trabecular bone parameters, including the volume bone mineral density (vBMD), tissue mineral density (TMD), trabecular bone volume fraction (BV/TV), trabecular number (Tb.N), trabecular thickness (Tb.Th), trabecular separation (Tb.Sp), structural model index (SMI), and connectivity density 
(Conn.D), were determined using TRI/3D-BON (Ratoc System Engineering Co., Tokyo, Japan) in accordance with the guidelines described by Bouxsein et al. [17].

\section{Micro-indentation testing for evaluation of bone strength}

Bone material strength was evaluated using micro-indentation[16, 18]. The specimens for mechanical analysis were embedded in epoxy resin, and 5-mm thick sections were cut. The bone-exposed surfaces of the resin-embedded specimens were polished and mirror-finished. The specimens were set on an indentation test machine equipped with a combination of a universal mechanical testing machine (Model 4411, Instron Corp., Norwood, MA, USA) and a microscope (VH5000, KEYENCE, Japan). The mechanical strength of three points in the cortical bone region of each specimen, the sites of which were confirmed under a microscope, was examined using a spherical indenter with a 500- $\mu$ m diameter.

An indentation test was carried out under the following conditions: indentation speed of $10 \mu \mathrm{m} / \mathrm{min}$, maximum indentation depth of $10 \mu \mathrm{m}$, and sampling time of $100 \mathrm{~ms}$. The samples were kelp in a most condition during the mechanical testing. After the load-displacement relationship was recorded, the contact stiffness was determined by calculating the slope during loading.

\section{Histology, histochemistry, and immunohistochemistry}

The specimens were fixed in paraformaldehyde and decalcified in $5 \%$ ethylenediaminetetraacetic acid. Paraffin-embedded sections (5- $\mu$ m thick) were assessed using hematoxylin and eosin staining. To evaluate osteoblast and osteoclastic activity, paraffin sections were examined for tissue non-specific alkaline phosphatase (TNAP) and tartrate-resistant acid phosphatase (TRAP) activity. To evaluate osteocyte activity, dewaxed paraffin sections were incubated with rabbit polyclonal antibody against dentin matrix protein 1 (DMP-1) (Code No. M176; TaKaRa Bio Inc., Otsu, Japan), followed by incubation with horse radish peroxidase-conjugated anti-rabbit IgG (Chemicon International Inc., Temecula, CA). For visualization of immunoreactions, diaminobenzidine tetrahydrochloride was used as a substrate. To evaluate osteocyte apoptosis, the ratio of empty lacunae was determined in a region of interest measuring $3200 \times 2300 \mu \mathrm{m}$. All sections were counterstained with methyl green and observed under a light microscope (Eclipse E800, Nikon Instruments Inc., Tokyo, Japan).

A part of each specimen was immersed in a half-strength Karnovsky fixative, dehydrated with ascending concentrations of acetone and embedded in epoxy resin (TAAB, Berkshire, UK). Undecalcified semi-thin sections were stained with toluidine blue and observed under a light microscope (Merck, Darmstadt, Germany). Von Kossa staining was also performed on semi-thin sections to observe bone mineralization on the trabecular bone surface[19].

\section{Statistical analysis}

Comparisons of the between-group data were performed using Student's t-test and Fisher's exact test, as appropriate. A P-value < 0.05 was considered significant for all comparisons. Data are presented as means \pm standard deviation (SD). Linear regression was used to calculate correlations between contact stiffness and other variables using Pearson's product-moment correlation coefficient, with the 
significance level set at $P<0.05$. All statistical analyses were performed using JMP Pro version 15 (SAS Institute Inc., Cary, NC, USA).

\section{Results}

\section{Demographic characteristics}

All patients in the GIOBP group received GCs and BP with a mean dosing period of 6.7 (SD 2.0 years, range 3-10 years). The mean age at biopsy in the GIOBP group was 67.6 years. All patients received disease-modifying antirheumatic drugs, and three patients were treated with biological agents.

The age and BMD of patients in the reference group were matched to those in the GIOBP group. A comparison of demographic data between the GIOBP and reference groups is shown in Table 1. There was no significant difference in the prevalence of coexisting illnesses, such as T2D and chronic kidney disease (CKD). Renal function (blood urea nitrogen, creatinine, and estimated glomerular filtration rate) or electrolytes ( $\mathrm{Ca}$ and inorganic phosphate) were not significantly different between the two groups. The DXA-evaluated BMD of the lumbar spine and femoral neck was comparable between the two groups. 
Table 1

Demographic characteristics.

\begin{tabular}{|c|c|c|c|}
\hline & GIOBP $(n=10)$ & Reference $(n=10)$ & P-values \\
\hline Age (years) & $67.6 \pm 8.7$ & $68.1 \pm 5.3$ & 0.879 \\
\hline $\mathrm{BMI}\left(\mathrm{kg} / \mathrm{mm}^{2}\right)$ & $24.0 \pm 4.8$ & $23.3 \pm 4.8$ & 0.764 \\
\hline Age at menopause & $49.6 \pm 1.9$ & $48.3 \pm 2.6$ & 0.216 \\
\hline \multicolumn{4}{|l|}{ Comorbidity (\%) } \\
\hline $\mathrm{T} 2 \mathrm{D}$ & $40 \%$ & $20 \%$ & 0.303 \\
\hline CKD $\geq$ stage 3 & $20 \%$ & $20 \%$ & 1.000 \\
\hline Smoking habits (\%) & $20 \%$ & $20 \%$ & 1.000 \\
\hline Drinking alcohol habits (\%) & $0 \%$ & $10 \%$ & 1.000 \\
\hline Parental history of hip fracture & $10 \%$ & $10 \%$ & 1.000 \\
\hline \multicolumn{4}{|l|}{ Blood tests } \\
\hline BUN (mg/dL) & $15.6 \pm 4.2$ & $14.9 \pm 4.4$ & 0.718 \\
\hline Cre $(\mathrm{mg} / \mathrm{dL})$ & $0.63 \pm 0.19$ & $0.65 \pm 0.11$ & 0.782 \\
\hline $\operatorname{eGFR}\left(\mathrm{ml} / \mathrm{min} / 1.73 \mathrm{~m}^{2}\right)$ & $76.9 \pm 22.5$ & $70.8 \pm 13.4$ & 0.473 \\
\hline $\mathrm{Ca}(\mathrm{mg} / \mathrm{dL})$ & $9.10 \pm 0.74$ & $9.00 \pm 0.74$ & 0.778 \\
\hline $\mathrm{IP}(\mathrm{mg} / \mathrm{dL})$ & $3.62 \pm 0.25$ & $3.73 \pm 0.39$ & 0.635 \\
\hline \multicolumn{4}{|l|}{$\operatorname{BMD}\left(\mathrm{g} / \mathrm{cm}^{2}\right)$} \\
\hline Lumbar spine & $0.85 \pm 0.16$ & $0.77 \pm 0.13$ & 0.201 \\
\hline Femoral neck & $0.61 \pm 0.08$ & $0.54 \pm 0.09$ & 0.289 \\
\hline \multicolumn{4}{|c|}{ Data are shown as means \pm standard deviations or as percentages. } \\
\hline \multicolumn{4}{|c|}{$\begin{array}{l}\text { T2D: type } 2 \text { diabetes mellitus; CKD: chronic kidney disease; eGFR: estimated glomerular filtration rate } \\
\text { BMD: bone mineral density BUN: blood urea nitrogen; Cre: creatinine; IP: inorganic phosphate. }\end{array}$} \\
\hline
\end{tabular}

The fracture severity index, which we defined as the sum of the semiquantitative grade of vertebral fractures, was significantly greater in the GIOBP group than in the reference group (Table 2).

According to the FRAX-based fracture risk estimation, major osteoporosis fracture risk and femoral neck fracture risk were three times higher in the GIOBP group than in the reference group, probably due to two 
positive items (RA and GCs). The major osteoporosis fracture risk score was moderately correlated with the fracture severity index $(r=0.590, P=00062)$.

Table 2

Comparison of estimated fracture risk, existing vertebral fractures, and contact stiffness of bone biopsy in the GIOBP group with the reference group.

\begin{tabular}{|c|c|c|c|}
\hline & GIOBP & Reference & P-values \\
\hline \multicolumn{4}{|c|}{ Fracture risk estimation by FRAX score } \\
\hline Major osteoporosis fracture (\%) & $33.4 \pm 8.0$ & $10.7 \pm 4.8$ & $<0.001$ \\
\hline Femoral neck fracture (\%) & $9.9 \pm 6.1$ & $2.8 \pm 2.4$ & 0.002 \\
\hline \multicolumn{4}{|l|}{ Existing vertebral fractures } \\
\hline Number of vertebral fractures & $2.0 \pm 1.6$ & $0.3 \pm 0.7$ & 0.007 \\
\hline Vertebral fracture severity index & $4.1 \pm 3.8$ & $0.3 \pm 0.7$ & 0.006 \\
\hline
\end{tabular}

FRAX: fracture risk assessment tool.

\section{Bone quality and mechanical strength in patients with RA receiving long-term GC and BP therapy}

Micro-CT evaluation was performed to assess the microstructure of the trabecular bone. Reconstructed three-dimensional micro-CT images of bone biopsy samples showed noticeable structural deterioration in the trabecular bone in the GIOBP group compared to the reference group (Fig. 1A). Quantitative analysis of the microstructure of trabecular bone showed that the GIOBP group had a significantly lower BV/TV, Tb.Th., Tb.N., and Conn.D, and a significantly higher Tb.Sp. and SMI compared with the reference group (Fig. 1B). TMD and VBMD in the GIOBP group were significantly lower than those in the reference group (Fig. 1C).

We next evaluated the actual mechanical strength of the bone biopsy specimen in each patient using micro-indentation testing (Fig. 2A, B, and C). The contact stiffness of the cortical iliac bone biopsy samples had a moderate negative correlation with the fracture severity index (Fig. 2D). The contact stiffness was significantly lower in the GIOBP group than in the reference group (GIOBP $164.9 \pm 36.5$ vs. reference $218.8 \pm 24.6, P=0.0014 ; F i g .2 E$ ). We further studied the correlation between contact stiffness and possible associated factors, including age, BMI, CKD stage, daily prednisolone dosage, and TMD (Fig. 2F). We found that the daily prednisolone dose was strongly negatively correlated with contact stiffness and that TMD was moderately positively correlated with contact stiffness. We also compared the effect of T2D and smoking on contact stiffness, but there were no significant differences in the contact stiffness between T2D and non-T2D patients ( $199.5 \pm 70.8$ vs. $191.03 \pm 20.1, P=0.701)$, and smokers and non-smokers ( $193.8 \pm 46.1$ vs. $191.1 \pm 4.92, \mathrm{P}=0.909)$. 
Underlying mechanisms responsible for bone quality deterioration in patients with RA receiving long-term GC and BP therapy

To gain insight into the mechanism of bone quality deterioration, serologic and histologic analyses were performed. Despite the trend toward decreasing serum BAP levels in the GIOBP group compared with the reference group, the difference was not statistically significant $(P=0.07 ; \mathrm{Fig}$. $3 \mathrm{~A})$. However, according to histological assessment (Figure 4, 5), TNAP expression on the trabecular bone surface was impaired in the GIOBP group compared with the reference group. In the GIOBP group, there was a thinner layer of TNAP-positive osteoblasts on the trabecular bone surface compared with the reference group.

Furthermore, von Kossa-stained semi-thin sections showed impaired mineralization of the osteoid of trabecular bone, which was frequently observed in the GIOBP group compared with the reference group.

The mean level of serum TRACP-5b was significantly lower in the GIOBP group compared to the reference group, suggesting that bone resorption was suppressed by chronic BP administration in patients with RA receiving GC therapy (Fig. 3B). TRAP-reactive osteoclasts were less likely to be observed on the surface of the trabecular bone in the GIOBP group compared with the reference group.

Histological assessment verified that lacunae empty of osteocytes were frequently observed in the GIOBP group while rarely seen in the reference group (Fig. 4, 5). High-magnification observations of toluidine blue-stained semi-thin sections revealed osteocyte atrophy and the disappearance of bone canaliculi in the GIOBP group. The ratio of empty osteocyte lacunae to total osteocyte lacunae was significantly higher in the GIOBP group than in the reference group (Fig. 3C). DMP-1 immunoreactivity was detected in osteocytes of the trabecular bone in the reference group (Fig. 4F); however, the GIOBP group did not show many osteocytes with an intense DMP-1 positivity (Fig. 5F).

\section{Discussion}

The results of this study provide supportive evidence for the concept that long-term BP administration has a minor benefit in the prevention of fragility fractures in GIO. This concept has been mentioned in several review papers $[8,9]$, but clinical evidence is difficult to obtain due to the infeasibility of a long-term placebo-controlled clinical trial for GIO. Since GIO inevitably increases fragility fracture risk, using a longterm placebo for these patients is unethical. Furthermore, thousands of patients are usually required to obtain evidence of fracture prevention. This study did not provide a definite answer to whether long-term $\mathrm{BP}$ therapy deteriorates bone quality in GIO because the reference patient group did not include GIO patients who had not received BP but age- and BMD-matched non-RA postmenopausal women. Nonetheless, our data suggest that long-term BP therapy is insufficient to decrease fragility fracture risk in the chronic stage of GC treatment.

The most important finding of this study is that patients with GIO receiving long-term BP therapy exhibited significantly lower bone material strength than age-matched postmenopausal women and that bone material strength was negatively correlated with vertebral fracture severity, consistent with a previous study $[16,20]$. These results do not mean that BP is ineffective for GIO but indicate that BP is 
insufficient to reduce the risk of GIO-induced fragility fracture. This notion is corroborated by the fact that the vertebral fracture severity score was significantly higher in the GIOBP group than in the reference group. Furthermore, it should be noted that bone material strength was negatively correlated with daily prednisolone dosage, suggesting that the deterioration of bone matrix quality greatly contributes to vertebral fragility in GIO even after long-term BP use.

Given that the degree of mineralization is a major intrinsic determinant of bone strength[21], our data suggest that low bone material strength in patients with RA receiving long-term BP therapy for $\mathrm{GIO}$ is attributable to the hypomineralized bone matrix. This idea is reinforced by our data showing a positive correlation between bone material strength and the degree of mineralization. Although there is no standard value for the degree of mineralization, the idea that patients in the GIOBP group had a low degree of mineralization can be justified by the fact that the majority of postmenopausal women have low bone matrix mineralization due to high bone turnover rates and that the degree of mineralization was significantly lower in the GIOBP group than in age-and BMD-matched postmenopausal patients. The hypomineralized bone matrix is probably due to the long-term impairment of osteoblastic activity by GC and $B P[10-12]$, but it is also partly attributable to impaired function and the apoptosis of osteocytes. Given that DMP-1 has been assumed to be an important regulator of biomineralization[22], reduced expression of DMP-1 in osteocytes in the GIOBP group may be associated with the hypomineralized bone matrix in patients with GIO receiving long-term BP. An earlier study showed that GCs induce the apoptosis of osteocytes, resulting in an enlargement of their lacunar space and the generation of a surrounding sphere of hypomineralized bone[23].

The compromised bone quality in patients with RA receiving long-term BP therapy for GIO is most likely attributable to their anti-remodeling effects on the low bone turnover form of osteoporosis (Fig. 6). In contrast to postmenopausal osteoporosis, which causes high bone turnover, chronic GC use induces low turnover osteoporosis by suppressing osteoclast activity and inhibiting osteoblast differentiation and function[10-12]. Given the pharmacological action of BP on bone metabolism, BP may further suppress bone remodeling and lead to severely suppressed bone turnover, resulting in adynamic bone and compromised bone quality in patients with GIO. In line with this idea, our histological data showed that bone resorption and bone formation were suppressed in patients with RA receiving an average of 6.7 years of BP treatment for GIO.

This study has some limitations, the most significant of which was the lack of appropriate controls. Earlier studies using iliac crest biopsies demonstrated low bone turnover in patients with non-GC treated RA compared to age- and sex-matched controls $[24,25]$. Although it is ideal to use patients with RA receiving GCs without osteoporosis medication as controls, we could not recruit such patients because $\mathrm{BP}$ is routinely administered for the prevention and treatment of $\mathrm{GIO}$ in line with the guidelines[3]. Second, we could not determine the number of fractures that occurred after commencing GC and BP therapies; fragility fractures may have occurred before receiving these treatments. We assessed the prevalent fractures using whole-spine plain X-ray films taken at the time of bone biopsy, but plain radiographs of the whole spine were not taken before commencing BP therapy. Most fragility fractures are 
asymptomatic and occur without major trauma, and therefore patients were not aware of them, so we could not determine whether fractures occurred before or after commencing BP therapy. Third, we did not evaluate other treatments for osteoporosis: additional studies are required to evaluate how bone formation agents, such as teriparatide and anti-sclerostin antibody, could affect bone quality in RA patients receiving GCs.

\section{Conclusions}

In conclusion, BP is routinely administered for the prevention and treatment of GIO regardless of the duration of GC administration, in line with GIO management guidelines. However, this study showed that rheumatoid arthritis patients who received long-term BP therapy for GIO exhibited compromised bone quality, possibly due to severely suppressed bone turnover, suggesting a minor benefit from long-term BP therapy for fracture prevention in the chronic stage of GC treatment.

\section{Abbreviations}

BAP

bone-specific alkaline phosphatase

BMD

bone mineral density

$\mathrm{BMI}$

body mass index

BP

bisphosphonate

BUN

blood urea nitrogen

$\mathrm{BV} / \mathrm{TV}$

bone volume/total volume

Conn.D

connective density

Cre

creatinine

T2D

type 2 diabetes mellitus

DMP-1

dentin matrix protein 1

DXA

dual-energy X-ray absorptiometry

eGFR

estimated glomerular filtration rate 
FRAX

Fracture Risk Assessment Tool

GC

glucocorticoid

GIO

glucocorticoid-induced osteoporosis

GIOBP

bisphosphonate therapy for glucocorticoid-induced osteoporosis

IP

inorganic phosphate

micro-CT

micro-computed tomography

RA

rheumatoid arthritis

SMI

structure model index

Tb.N

trabecular number

Tb.Sp

trabecular separation

Tb.Th

trabecular thickness

TMD

tissue mineral density

TNAP

tissue non-specific alkaline phosphatase

TRAP

tartrate-resistant acid phosphatase

vBMD

volumetric bone mineral density.

\section{Declarations}

\section{Ethics approval and consent to participate}

This study was approved by the Hokkaido University Hospital Institutional Review Board (\#012-0023) and written informed consent was obtained from all study participants.

Consent for publication

Availability of data and materials 


\section{Competing interests}

All authors state that they have no conflicts of interest.

\section{Funding}

This project was supported in part by a Grant-in-Aid for Scientific Research (C) from the Ministry of Education, Culture, Sports, Science, and Technology of Japan 25462357 (M. Takahata).

\section{Authors' contributions}

MT: research design, interpretation of data, and drafting and critically revising the paper.

TS: acquisition, analysis, and interpretation of data, and drafting the paper.

SY: acquisition and analysis of data

TY: acquisition and analysis of data

TH: acquisition and analysis of data, interpretation of data

RF: acquisition and analysis of data

HK: analysis of data

TE: analysis of data

YK: analysis of data

NA: interpretation of data

MT: acquisition and analysis of data

JO: acquisition and analysis of data

TK: acquisition and analysis of data

$\mathrm{NI}$ : interpretation of data and critically revising the paper

All authors have read and approved the final submitted manuscript.

\section{Acknowledgments}

This project was supported in part by a Grant-in-Aid for Scientific Research (C) from the Ministry of Education, Culture, Sports, Science, and Technology of Japan 25462357 (M. Takahata). 


\section{References}

1. Amiche MA, Albaum JM, Tadrous M, Pechlivanoglou P, Lévesque LE, Adachi JD, et al. Fracture risk in oral glucocorticoid users: a Bayesian meta-regression leveraging control arms of osteoporosis clinical trials. Osteoporosis Int. 2016;27:1709-18.

2. Nampei A, Hashimoto J, Koyanagi J, Ono T, Hashimoto $H$, Tsumaki N, et al. Characteristics of fracture and related factors in patients with rheumatoid arthritis. Mod Rheumatol. 2008;18:170-6.

3. Buckley L, Guyatt G, Fink HA, Cannon M, Grossman J, Hansen KE, et al. 2017 American College of Rheumatology Guideline for the Prevention and Treatment of Glucocorticoid-Induced Osteoporosis. Arthritis Rheumatol. 2017;69:1521-37.

4. Saag KG, Emkey R, Schnitzer TJ, Brown JP, Hawkins F, Goemaere S, et al. Alendronate for the Prevention and Treatment of Glucocorticoid-Induced Osteoporosis. New Engl J Medicine. 1998;339:292-9.

5. Saag KG, Shane E, Boonen S, Marín F, Donley DW, Taylor KA, et al. Teriparatide or Alendronate in Glucocorticoid-Induced Osteoporosis. New Engl J Medicine. 2007;357:2028-39.

6. Sambrook PN, Kotowicz M, Nash P, Styles CB, Naganathan V, Henderson-Briffa KN, et al. Prevention and Treatment of Glucocorticoid-Induced Osteoporosis: A Comparison of Calcitriol, Vitamin D Plus Calcium, and Alendronate Plus Calcium. J Bone Miner Res. 2003;18:919-24.

7. Wang Y-K, Zhang Y, Qin S-Q, Wang X, Ma T, Guo J-B, et al. Effects of alendronate for treatment of glucocorticoid-induced osteoporosis: A meta-analysis of randomized controlled trials. Medicine. 2018;97:e12691.

8. Lems WF, Saag K. Bisphosphonates and glucocorticoid-induced osteoporosis: cons. Endocrine. 2015;49:628-34.

9. Teitelbaum SL, Seton MP, Saag KG. Should bisphosphonates be used for long-term treatment of glucocorticoid-induced osteoporosis? Arthritis Rheumatism. 2011;63:325-8.

10. Takahata M, Maher JR, Juneja SC, Inzana J, Xing L, Schwarz EM, et al. Mechanisms of bone fragility in a mouse model of glucocorticoid-treated rheumatoid arthritis: Implications for insufficiency fracture risk. Arthritis Rheumatism. 2012;64:3649-59.

11. Yao W, Cheng Z, Busse C, Pham A, Nakamura MC, Lane NE. Glucocorticoid excess in mice results in early activation of osteoclastogenesis and adipogenesis and prolonged suppression of osteogenesis: A longitudinal study of gene expression in bone tissue from glucocorticoid-treated mice. Arthritis Rheumatism. 2008;58:1674-86.

12. Weinstein RS, Jilka RL, Parfitt AM, Manolagas SC. Inhibition of osteoblastogenesis and promotion of apoptosis of osteoblasts and osteocytes by glucocorticoids. Potential mechanisms of their deleterious effects on bone. J Clin Invest. 1998;102:274-82.

13. Staa TPV, Leufkens HGM, Abenhaim L, Zhang B, Cooper C. Use of Oral Corticosteroids and Risk of Fractures. J Bone Miner Res. 2000;15:993-1000.

14. Weinstein RS. Glucocorticoid-Induced Bone Disease. New Engl J Medicine. 2011;365:62-70. 
15. Genant HK, Wu CY, Kuijk C van, Nevitt MC. Vertebral fracture assessment using a semiquantitative technique. J Bone Miner Res. 1993;8:1137-48.

16. Sosa DD, Eriksen EF. Reduced Bone Material Strength is Associated with Increased Risk and Severity of Osteoporotic Fractures. An Impact Microindentation Study. Calcified Tissue Int. 2017;101:34-42.

17. Bouxsein ML, Boyd SK, Christiansen BA, Guldberg RE, Jepsen KJ, Müller R. Guidelines for assessment of bone microstructure in rodents using micro-computed tomography. J Bone Miner Res. 2010;25:1468-86.

18. Herrera S, Diez-Perez A. Clinical experience with microindentation in vivo in humans. Bone. 2017;95:175-82.

19. Yamamoto T, Hasegawa T, Sasaki M, Hongo H, Tsuboi K, Shimizu T, et al. Frequency of Teriparatide Administration Affects the Histological Pattern of Bone Formation in Young Adult Male Mice. Endocrinology. 2016;157:2604-20.

20. Mellibovsky L, Prieto-Alhambra D, Mellibovsky F, Güerri-Fernández R, Nogués X, Randall C, et al. Bone Tissue Properties Measurement by Reference Point Indentation in Glucocorticoid-Induced Osteoporosis. J Bone Miner Res. 2015;30:1651-6.

21. Boivin G, Bala Y, Doublier A, Farlay D, Ste-Marie LG, Meunier PJ, et al. The role of mineralization and organic matrix in the microhardness of bone tissue from controls and osteoporotic patients. Bone. 2008;43:532-8.

22. Feng JQ, Ward LM, Liu S, Lu Y, Xie Y, Yuan B, et al. Loss of DMP1 causes rickets and osteomalacia and identifies a role for osteocytes in mineral metabolism. Nat Genet. 2006;38:1310-5.

23. Lane NE, Yao W, Balooch M, Nalla RK, Balooch G, Habelitz S, et al. Glucocorticoid-Treated Mice Have Localized Changes in Trabecular Bone Material Properties and Osteocyte Lacunar Size That Are Not Observed in Placebo-Treated or Estrogen-Deficient Mice. J Bone Miner Res. 2006;21:466-76.

24. Compston JE, Vedi S, Croucher PI, Garrahan NJ, O’Sullivan MM. Bone turnover in non-steroid treated rheumatoid arthritis. Ann Rheum Dis. 1994;53:163.

25. Mellish RW, O'Sullivan MM, Garrahan NJ, Compston JE. Iliac crest trabecular bone mass and structure in patients with non-steroid treated rheumatoid arthritis. Ann Rheum Dis. 1987;46:830.

\section{Figures}



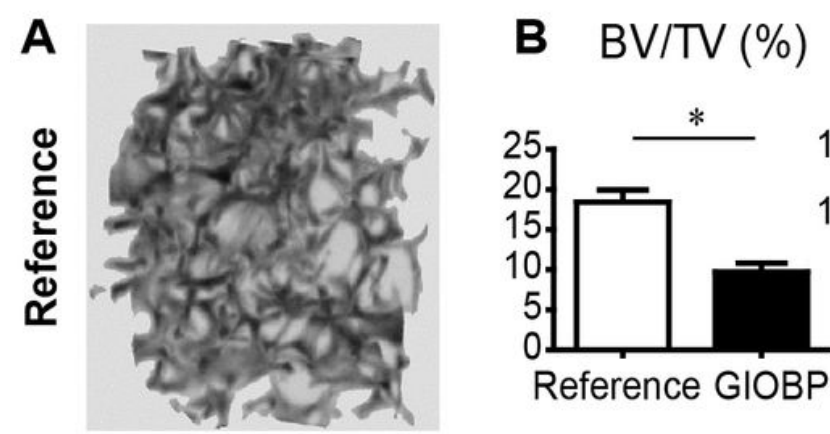

Tb.Th. $(\mu \mathrm{m})$

Tb.N. $(1 / \mathrm{mm})$

C $\operatorname{vBMD}\left(\mathrm{mg} / \mathrm{cm}^{3}\right)$
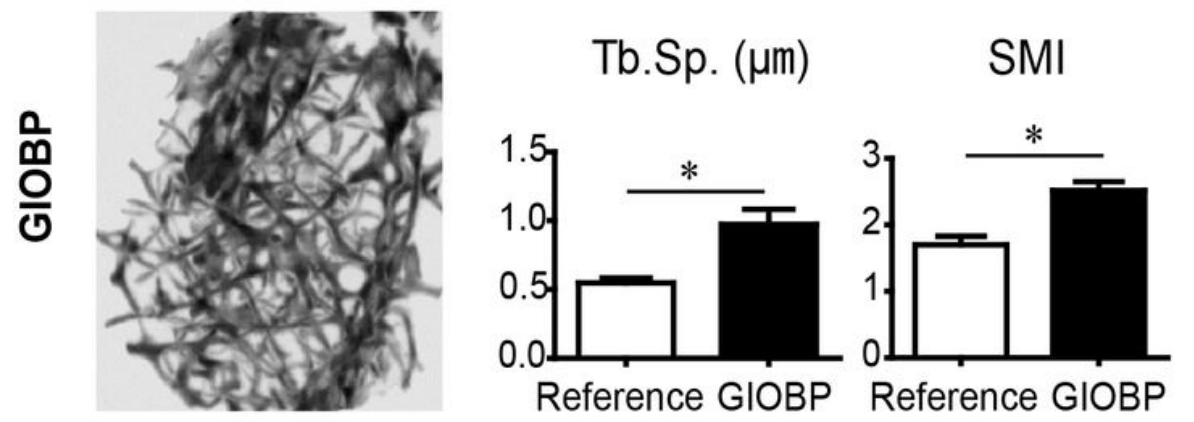

Conn.D. $\left(1 / \mathrm{mm}^{3}\right)$

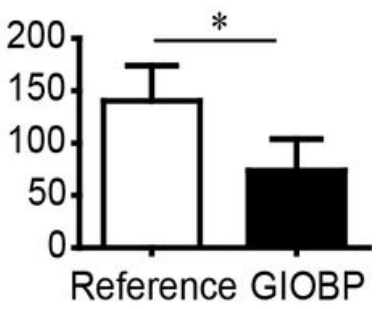

Reference GIOBP
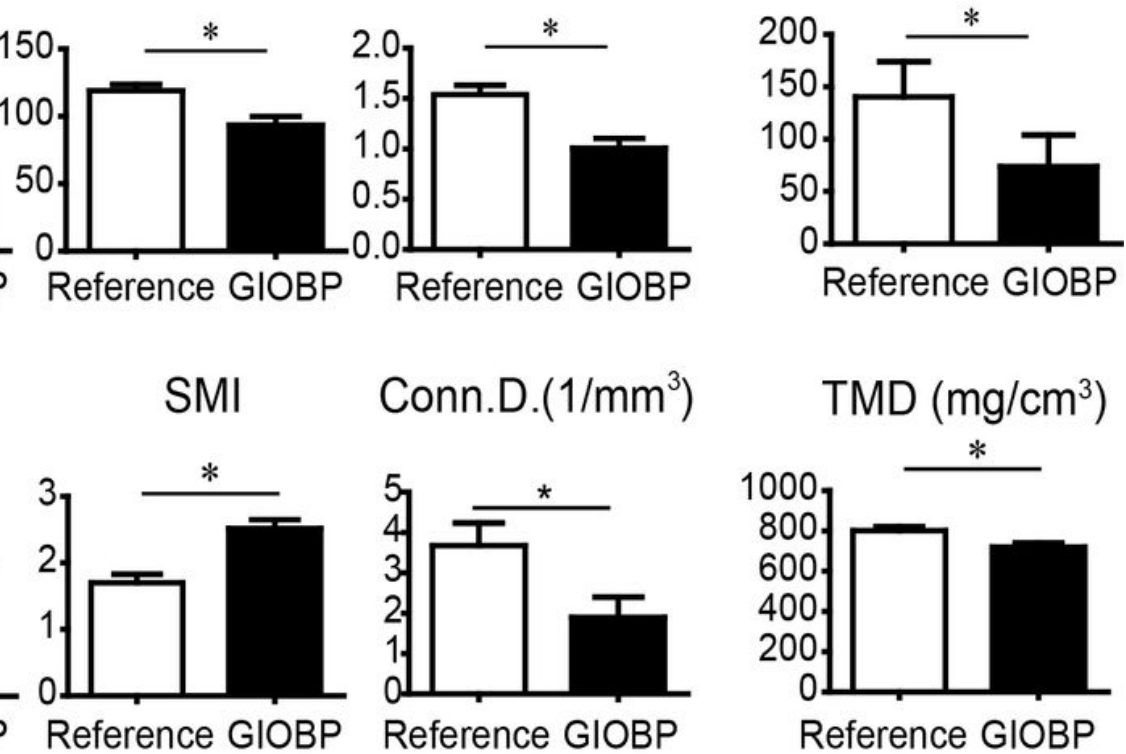

Figure 1

Microstructure analysis of iliac bone biopsy specimens as assessed by micro-CT. (A) Representative micro-CT images of the trabecular iliac bone biopsy specimens from the reference and GIOBP groups. (B) Comparisons of bone volume and microstructural indices of trabecular bone specimens between the reference and GIOBP groups. BV/TV, bone volume/total volume; Tb.Th, trabecular thickness; Tb.N, trabecular number; Tb.Sp, trabecular separation; SMI, structure model index; and Conn.D., connective density. (C) Comparisons of volumetric bone mineral density (vBMD) and tissue mineral density (TMD) of trabecular bone between the reference and GIOBP groups. The values shown are means \pm standard deviation $(S D)(* p<0.05)$. 


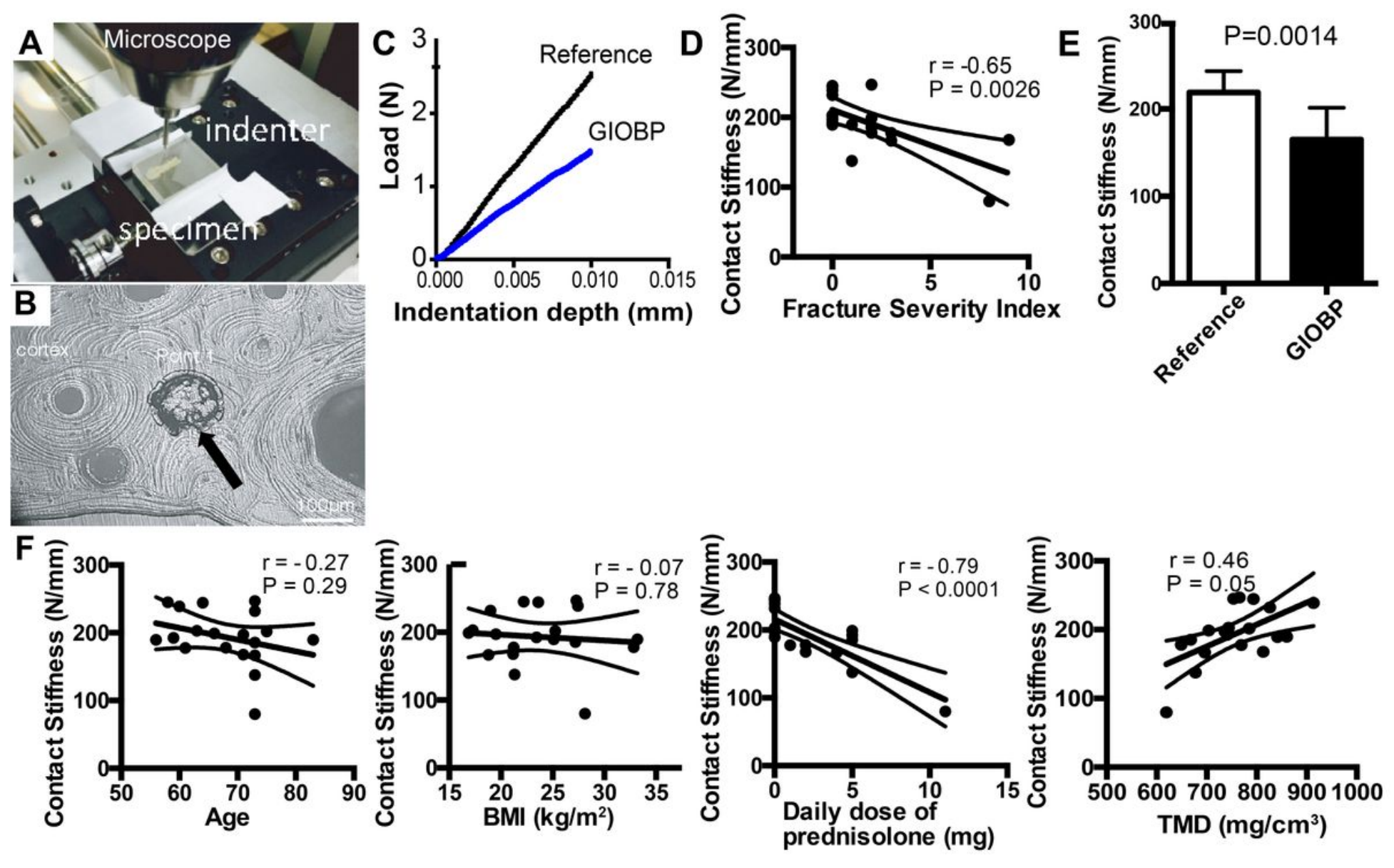

Figure 2

Bone material strength of iliac bone biopsy specimens as assessed by micro-indentation testing. (A) The indentation-testing device comprised a stage with a controller and microscope in a universal testing machine. The specimen could be moved and positioned for the indentation test under microscopy guidance. (B) Microscopic image after the indentation test is shown. The indenter's tip was soaked in India ink before testing to confirm the indentation point after testing (arrow). The indentation point was placed away from the Haversian canal. (C) Representative load-deformation curves from the reference and GIOBP groups. (D) The correlation between contact stiffness and fracture severity index. The scatter plot shows a negative linear correlation between contact stiffness and fracture severity index. The solid linear and curved lines indicate the regression line and 95\% confidence interval lines, respectively. (E) Comparison of contact stiffness between the reference and GIOBP groups. (F) The correlation between contact stiffness and age, body mass index (BMI), the daily dosage of prednisolone, and tissue mineral density (TMD). 
A

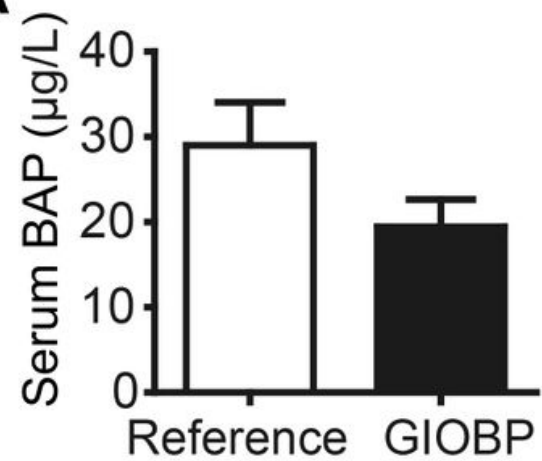

B

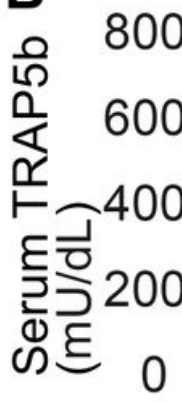

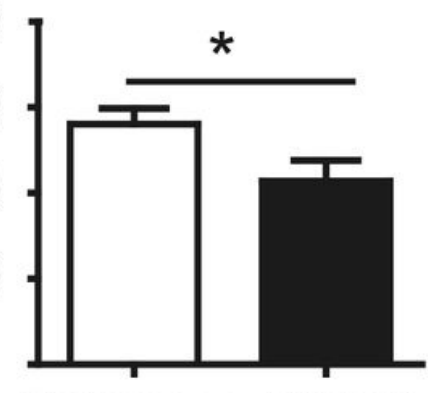

Reference GIOBP
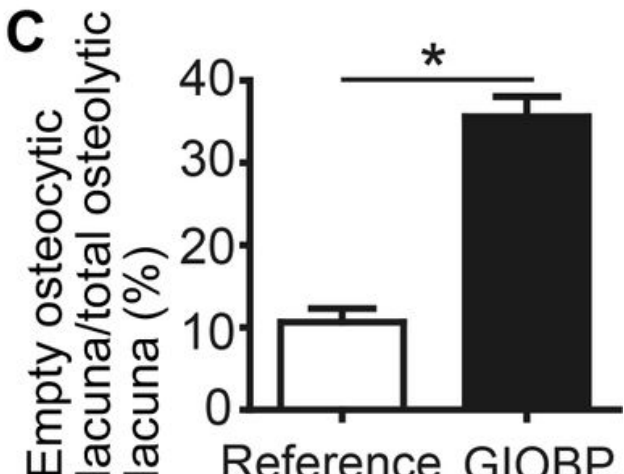

Reference GIOBP

\section{Figure 3}

Serum bone turnover markers and histomorphometric analysis of osteocytes (A, B) Comparison of serum bone-specific alkaline phosphatase (BAP) and tartrate-resistant acid phosphatase $5 b$ (TRACP-5b). (C) Comparison of the ratio of empty osteocytic lacunae to total osteocytic lacunae on histology. The values shown are means $\pm S D(* p<0.05)$.

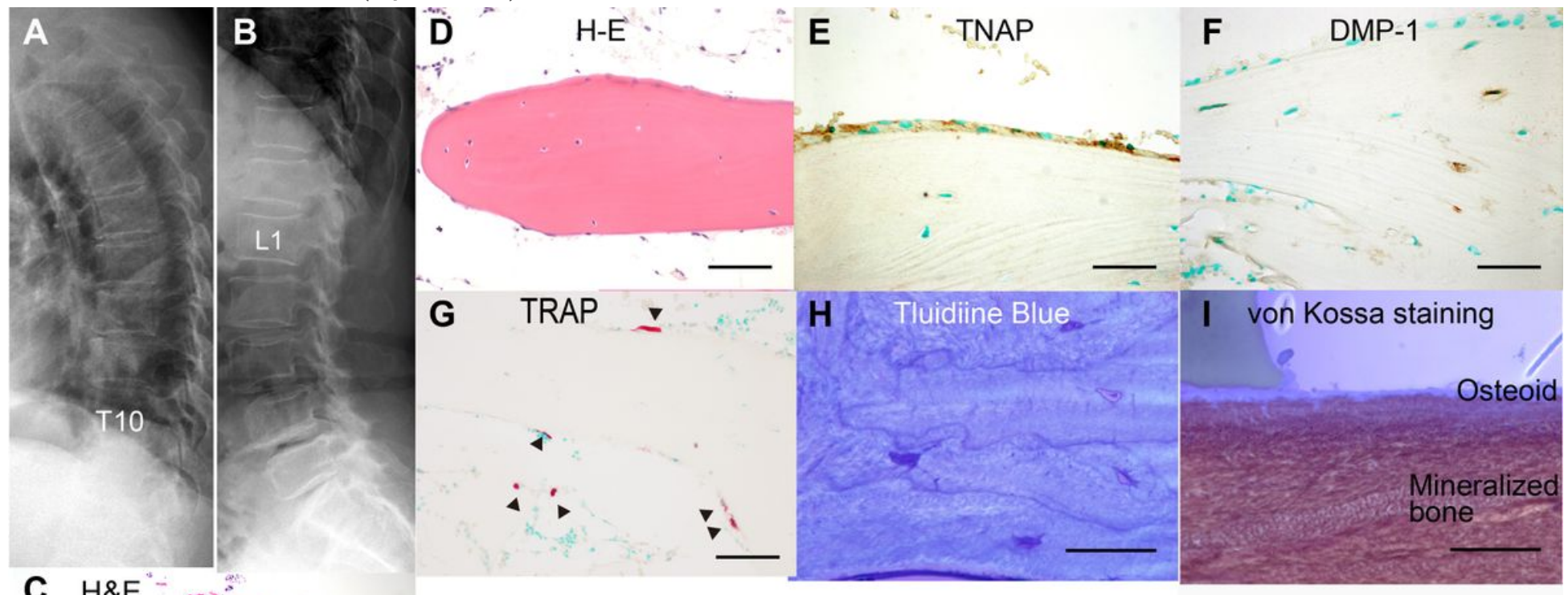

C H\&E

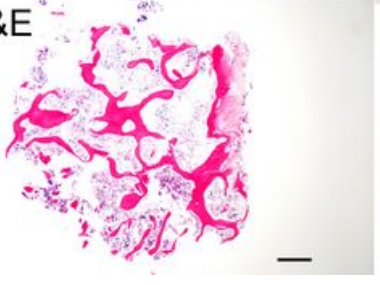

Figure 4

Representative case of a 73-year-old woman from the reference group. Plain lateral radiograms of the thoracic spine (A) and lumbar spine (B). (C-I) Representative micrographs of iliac bone biopsy specimen with hematoxylin and eosin (H-E), tissue non-specific alkaline phosphatase (TNAP), dentin matrix protein (DMP-1), tartrate-resistant acid phosphatase (TRAP), Toluidine blue, and von Kossa staining. Red stained cells (black arrowheads) on TRAP-stained sections indicate osteoclasts. Bars: C $=1000 \mu \mathrm{m} ; \mathrm{D}, \mathrm{G}=100$ $\mu \mathrm{m} ; \mathrm{E}, \mathrm{F}=50 \mu \mathrm{m} ; \mathrm{H}, \mathrm{I}=10 \mu \mathrm{m}$. 


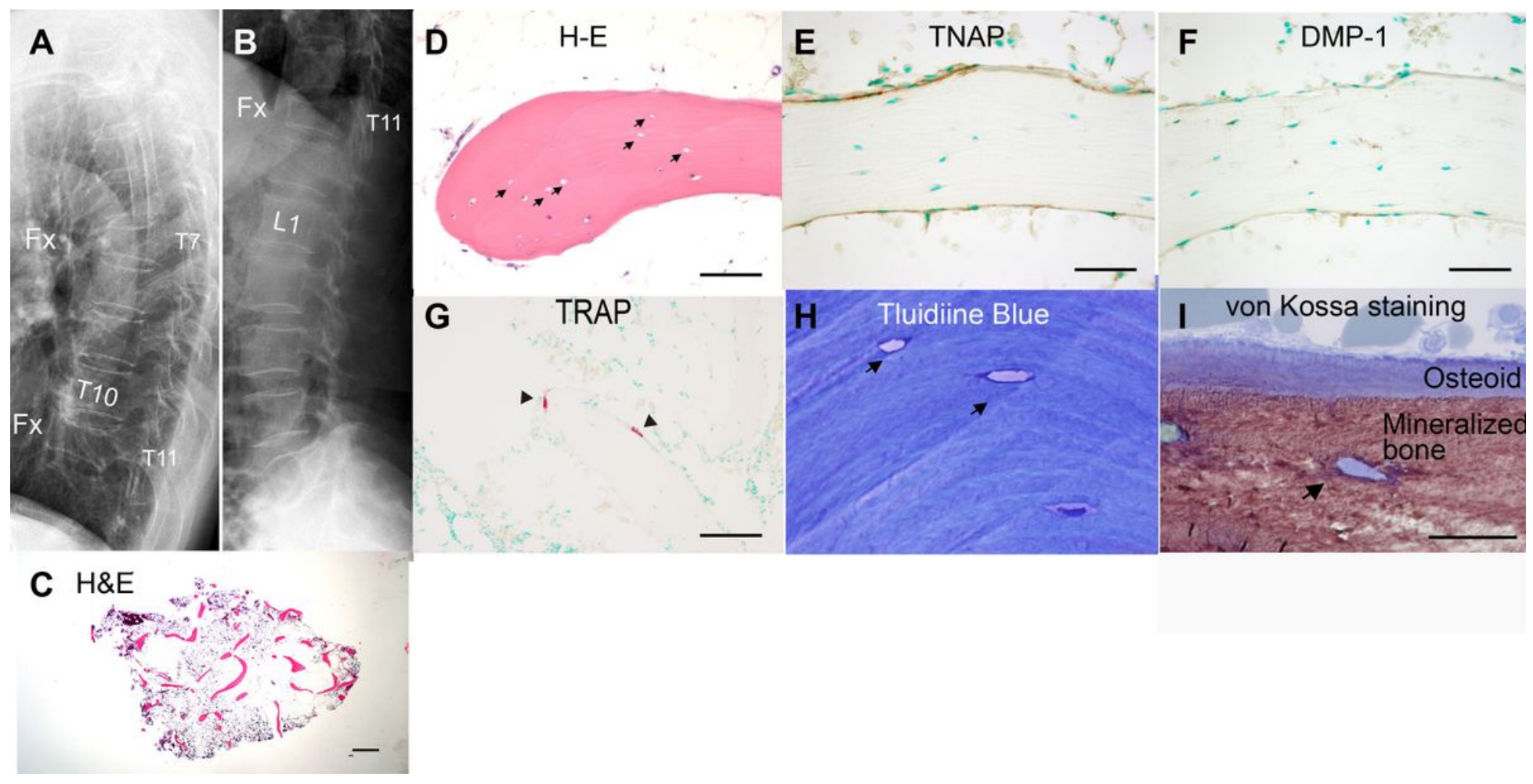

Figure 5

Representative case of a 73-year-old woman from the GIOBP group. Plain lateral radiograms of the thoracic spine (A) and lumbar spine (B) showed Genant grade 1 mild vertebral body compression fractures (Fx) at T7 and T11. (C-I) Representative micrographs of iliac bone biopsy specimen with hematoxylin and eosin (H-E), tissue non-specific alkaline phosphatase (TNAP), dentin matrix protein (DMP-1), tartrate-resistant acid phosphatase (TRAP), Toluidine blue, and von Kossa staining. Red stained cells (black arrowheads) on TRAP-stained sections indicate osteoclasts. Black arrows indicate empty osteocyte lacunae. Bars: $\mathrm{C}=1000 \mu \mathrm{m} ; \mathrm{D}, \mathrm{G}=100 \mu \mathrm{m} ; \mathrm{E}, \mathrm{F}=50 \mu \mathrm{m} ; \mathrm{H}, \mathrm{I}=10 \mu \mathrm{m}$. 


\section{Glucocorticoids}
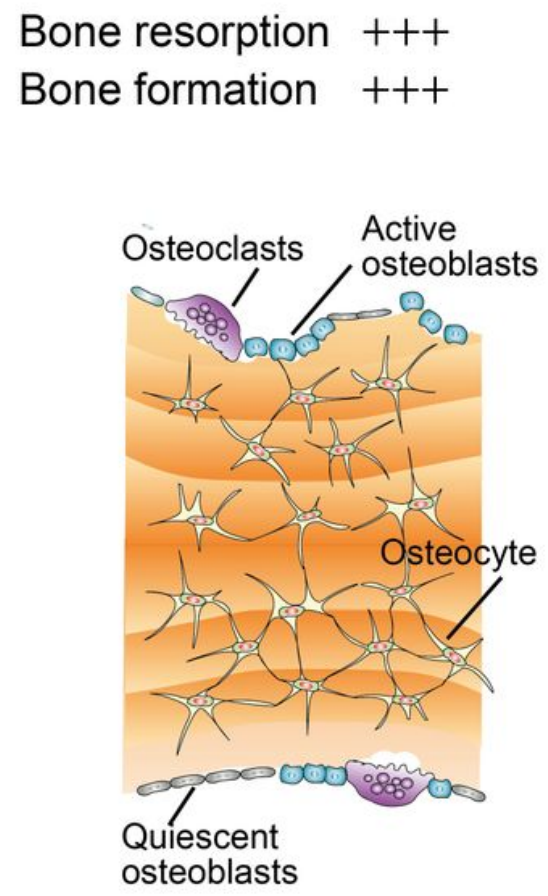
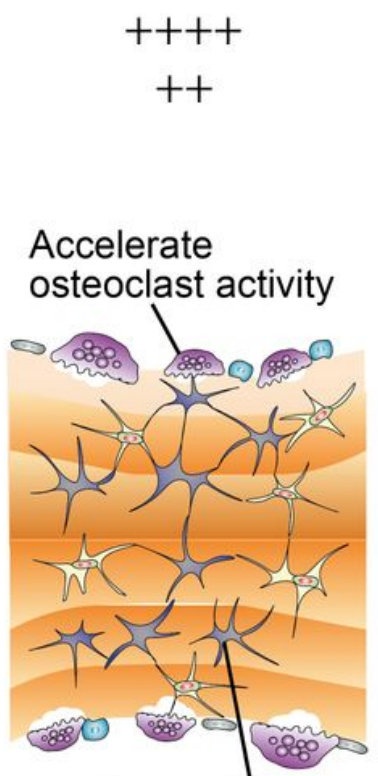

Induce the apoptosis of osteocytes

Suppress osteoblast activity
Glucocorticoids

Bisphosphonate

(Short term 1-2 years)

Glucocorticoids Bisphosphonate (Long term )

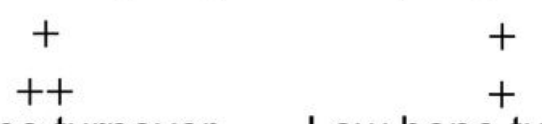

Low bone turnover Low bone turnover (Resorption < Formation) (Resorption >? Formation)

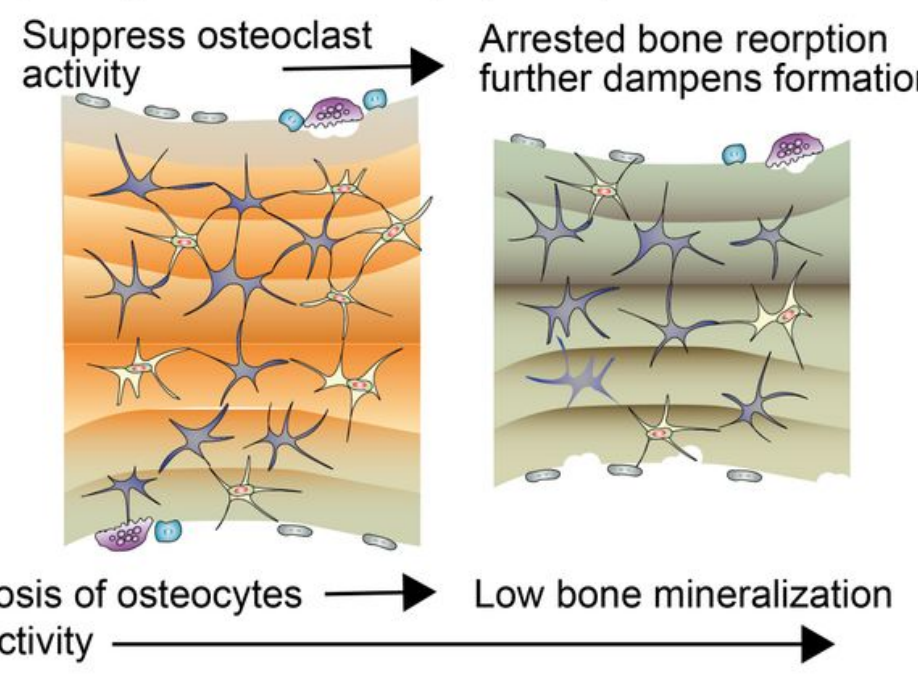

\section{Figure 6}

Schematic representation of the mechanism of action of glucocorticoids (GCs) and bisphosphonates (BP) for bone. GCs promote osteoclast activity, suppress osteoblast activity, and induce osteocyte apoptosis. BP treatment induces apoptosis of osteoclasts, leading to an increase in bone volume in the short term. Long-term administration of BP and GCs further slows bone turnover, with a disproportionate decrease in bone formation over a reduction in bone resorption, leading to deterioration of bone quality and quantity. 